\title{
Comet collision boosts controversy over global protection strategy
}

Washington. The greater than expected impact of the comet Shoemaker-Levy 9 as it crashed into Jupiter this week is set to intensify the debate over what steps, if any, should be taken to deal with the prospects of

Many astronomers are keen to proceed with an extensive survey of those comets and asteroids which are likely to infringe on the Earth's orbit, along the lines of the $\$ 250$ million Spaceguard proposal made two years ago by a panel set up by the National Aeronautics and Space Administration (NASA) - and as yet unfunded.

But some fear that the perceived threat is being overstated. In a strongly worded article in this summer's issue of the journal of Cornell University and Steven Ostro of NASA's Jet Propulsion Laboratory play down the risk.

Using a rough-and-ready risk analysis, they estimate that global impact catastrophes, when averaged out statistically, could account for 3,000 deaths per year. But they argue that this is insignificant when compared, for example, to the three million deaths a year from smoking, or those in developing countries dying from poverty and disease.

While supporting the Spaceguard proposal, Sagan and Ostro say they are alarmed at talk about the need for a nuclear weapons system to protect the planet from galactic debris. They warn that such a proposal is finding favour in "some elements of the defence establishment", even though such a weapons system is "well beyond the current economic capability of the planet, and would such an object striking the Earth. Issues in Science and Technology, Carl Sagan

introduce new risks that seem still more unacceptable".

The Spaceguard proposal would involve building and connecting six 2.5-metre diameter ground-based telescopes for $\$ 50$ million, and operating them at a cost of $\$ 10$ million a year for 20 years in order to survey asteroids crossing the Earth's trajectory and comets of more than one kilometre diameter.

Advocates say the survey would find 95 per cent of the asteroids, although they admit that its effectiveness in finding comets - which can arrive from anywhere in interstellar space - would be less easy to calculate. Some astronomers privately contend that the job could be done more cheaply with existing equipment.

Astronomers said this week that the Jupiter incident is likely to bring new impetus to the Spaceguard proposal. "It doesn't change the odds of a collision with Earth, but it makes the prospect more real," says Clark Chapman of the Planetary Science Institute, Tucson, Arizona. Paul Weissman of the Jet Propulsion Laboratory agrees. "It has demonstrated to people that comets do run into planets," he says.

The series of collisions with Jupiter particularly that of the largest part, fragment $\mathrm{H}$, which hit the planet on Monday after-

\section{LHC delay hits Japan's physicists}

Tokyo. Last month's failure by member states of the European Laboratory for Particle Physics (CERN) to agree on proceeding with plans for the Large Hadron Collider (LHC) has been a major setback for highenergy physicists in Japan who are trying to win their government's support for the proposed accelerator.

Japan's High Energy Committee, the top representative body of the high-energy physics community, decided late last year to throw its weight behind the LHC following the cancellation of the US Superconducting Super Collider (SSC) project. And the Ministry of Education, Science and Culture (MESC), which funds most of Japan's highenergy physics, is supporting the committee's position.

Officials from the ministry had been looking for a way to respond to requests from CERN for Japanese financial support for the European laboratory (see Nature 361, 288; 1993). Anticipating a clear decision in Geneva, they had been planning to make a budget request to the Ministry of Finance for the LHC in their budget proposals for fiscal year 1995 (which begins on 1 April), to be submitted at the end of August.

But the failure of CERN's council to reach agreement means that "we missed the chance", says Hirotaka Sugawara, director general of Japan's National Laboratory for High Energy Physics (KEK). Official approval by CERN's council was "crucial" for MESC to win approval from the Finance Ministry, says Sugawara. "Now we will have to wait until at least next year".

David Swinbanks

\author{
IMAGE \\ UNAVAILABLE \\ FOR COPYRIGHT \\ REASONS
}

\begin{abstract}
A series of infrared images in the K-band (2.2. $\mu \mathrm{m})$, taken at one minute intervals on the evening of 16
by the 0.75 metre telescope at the South African Astronomical Observatory, shows a flare caused by the first comet fragment (bottom left) increasing rapidly in and then decaying slowly.
\end{abstract}

noon with a vast explosion - - told astronomers at once that the density of the fragments was substantial, and that they had not disintegrated into a shoal of smaller meteorites as some had predicted.

Peter Leonard of the University of Maryland says that the density of comets had previously been estimated at between 0.2 and 0.9 that of water, the approximate densities of snow and ice respectively. But he estimated on Monday that the density of the fragment was around 0.5 _ "like snow with dust in it, and well held together".

Several physicists said they were surprised at the scale of the observations they have been able to make, after many had predicted that the collision might not live up to earlier expectations.

David Crisp of the NASA Jet Propulsion Laboratory, the principal investigator of the team at the Anglo-Australian Observatory at the Siding Spring Observatory site in New South Wales, Australia, said the event had exceeded his expectations "many times over".

The team was viewing Jupiter with the observatory's 3.90-metre reflecting telescope using a near infrared camera, as the two sub-fragments of what was originally fragment $\mathrm{G}$ collided on Monday morning. According to Crisp, the smaller fragment produced a fairly weak flash, about twice as bright as those produced by fragments $\mathrm{A}$ and C. "Then at about 7.40 all hell broke loose when the larger fragment hit."

Colin Macilwain \& Maggie Verrall 